\title{
A NEURAL NETWORK TO CORRECT MASS FLOW ERRORS CAUSED BY TWO-PHASE FLOW IN A DIGITAL CORIOLIS MASS FLOWMETER
}

\author{
R.P. Liu, M.J.Fuent ${ }^{\dagger}$, M.P. Henry, M. D. Duta \\ Engineering Science Department, Oxford University, Parks Road, Oxford OX1 3PJ, UK. \\ † Systems Engineering and Automatic Department, Facultad de Ciencias Universidad de \\ Valladolid.
}

\author{
Corresponding Author: renping.liu@eng.ox.ac.uk
}

\begin{abstract}
Coriolis mass flow meters provide accurate measurement of single-phase flows, typically to $0.2 \%$. However gas-liquid two-phase flow regimes may cause severe operating difficulties as well as measurement errors in these flow meters. As part of the Sensor Validation (SEVA) research at Oxford University a new fully digital coriolis transmitter has been developed which can operate with highly aerated fluids. This paper describes how a neural network has been used to correct the mass flow measurement for two-phase flow effects, based entirely on internally observed parameters, keeping errors to within $2 \%$. The correction strategy has been successfully implemented on-line in the coriolis transmitter. As required by the SEVA philosophy, the quality of the corrected measurement is indicated by the on-line uncertainty provided with each measurement value.
\end{abstract}

\section{INTRODUCTION}

A coriolis mass flow meter consists of a vibrating flowtube and an electronic transmitter to maintain vibration and to perform measurement calculations (Figure 1). Flowtube designs vary widely (e.g. straight-through pipes, U-tubes, B-tubes, with single path or split flow configurations), but the basic operation remains the same: mass flow through a vibrating tube causes a proportional coriolis force to act, which is detected as a phase difference between two velocity sensors. Almost all transmitter designs cause the flowtube to vibrate at its natural frequency; this frequency is a function of the process fluid density, which can thus be calculated and given as an additional measurement. The process fluid temperature is also monitored, to provide temperature correction of the mass flow and density (compensating for tube stiffness), and as an extra process measurement. The coriolis meter is thus a fairly sophisticated example of a multivariable industrial sensor.

Coriolis meters offer several advantages over other flowmeter technologies, such as accuracy and repeatability of $0.2 \%$ or better, and the ability to deal with difficult, non-Newtonian fluids such as slurries and foodstuffs. There are also disadvantages. The most serious is high cost, but another is the impact of two-phase (gas-liquid) flow on meter performance. Even a short burst of gas in a liquid flow stream (due for example to cavitation) may cause serious disruption to meter operation and lead to large measurement errors, as shown by recently published experiment work (Skea and Hall 1999).

The Engineering Science Department at the University of Oxford has been carrying out research into Self-Validation (SEVA) Sensors (Henry and Clark, 1993) since 1988, with funding from industry and the UK government. A number of prototype SEVA sensors have been developed based upon commercial devices. The coriolis meter was the basis of the first prototype. One benefit of carrying out a sensor validation analysis on the coriolis flowmeter is that it can lead to the redesign of the transmitter. The identification of the major limitations and fault modes of an instrument, and their impact on measurement quality, provides strong motivation for improvements in the basic design. As a consequence of validation work, a new prototype transmitter was designed in which all the analogue circuitry has been replace by digital components. This is illustrated in fig 2. A form of digital, Fourier- 
based signal processing, which is effectively independent of the signal amplitude, was used, as outlined in (Henry et al., 2000).

Note that throughout this paper comparisons are made between the new digital prototype transmitter and the current commercial, and mostly analogue, transmitter, driving in either case the same commercial flowtube. No attempt is made to compare with other commercial products. For example, some commercial transmitters have included digital components since 1990.

The benefits provided by the digital transmitter include: improvement in the measurement precision, improved flowtube control especially in response to two-phase flow, reduced influence of transmitter hardware on measurement data, allowing new research into the effects of two-phase flow, techniques for fault detection, and so on.

Although the digital transmitter is able to maintain flowtube oscillation at any level of two-phase flow (Henry et al, 2000), mass flow errors are still induced. In this paper, the twophase flow performance of the Coriolis flow meter is studied, and a method to predict the mass flow error using a neural network is proposed. Finally the results of the on-line implementation of the neural network for mass flow correction are presented.

\section{TWO-PHASE FLOW MEASUREMENT IN CORIOLIS FLOW METERS}

Two-phase flow measurement is carried out using a variety of instrumentation. Reimann (1989) reviews a variety of technologies for two-phase flow measurement, such as nuclear densitometry, tomography, and neutron scattering. Coriolis metering, which is relatively inexpensive compared to these technologies, has rarely been considered viable. Early studies of the effects of two-phase flow on coriolis metering demonstrated that meters operation was not reliable. Grumski and Bajura (1984) carried out an extensive two-phase flow (air with water, air with ethylene glycol) laboratory study with two types of early coriolis flowmeter. Attempts to model the measurement errors induced by two-phase flow were not very successful. Recently, Skea and Hall (1999) carried out experimental work on two-phase flow in coriolis flowmeters. The measurement was limited to gas fractions of $6 \%$ and $9 \%$. It is assumed by the current authors that at higher gas fractions the coriolis meters were unable to operate. They experienced difficulties in obtaining a stable signal from the meter pickups, due to the instability of oscillation of the tube under two-phase flow. Skea and Halls' results for two-phase flow (gas-in-oil) show that the measurement errors have poor consistency for both straight-tube and U-tube coriolis flowtube designs.

Hemp and Sultan (1989) carried out a theoretical study and modelling of two-phase flow in a coriolis flow meter. The 'bubble' or 'effective mass' model was described. It assumes that at small void fraction the behaviour of two-phase flow can largely be explained by considering the effective mass of a bubble in a liquid. A small sphere (or bubble) of low density (gas) is surrounded by fluid of high density. If both are subject to an acceleration (for example in a vibrating tube), then the bubble moves within the fluid, causing a drop in the observed inertia of the whole system. Defining the void fraction $\alpha$ as the proportion of gas by volume, then the effective mass drops by a proportion $\mathrm{R}$, with

$$
\mathrm{R}=\frac{2 \alpha}{1-\alpha}
$$

However, the actual response observed from a commercial meter was very different from this model. Since then there has been very little academic investigation of the problem.

Validation work on a commercial transmitter at Oxford has indicated the influence of transmitter hardware and software limitations on the two-phase flow response. The design of the digital transmitter was motivated in part by the desire to remove these influences, so that what was observed was primarly due to the physical interaction of flowtube and two-phase flow, and not due to transmitter limitations.

With the digital transmitter, the two-phase flow measurement error is roughly correlated with the 'bubble' model. Figure 3 shows the observed mass-flow errors for a series of runs at different flow rates. The dashed line shows the relationship between mass-flow error and density drop as predicted by the bubble model. Note that with the commercial transmitter the mass-flow error curves are quite different, and with a density drop greater than $2-5 \%$, the analog transmitter ceases to function entirely. Thus fig. 3 already represents a significant improvement in performance over the commercial transmitter. At high flows, only lower density drop data is shown. This is due to the limit on the air pressure supply and not on 
the ability of the flowmeter to maintain operation.

Again, it is important to point out that these results are only valid for the specific flowtube and transmitter designs. For example, although many other commercial designs stall at low void fractions, some are able to maintain vibration up to $40 \%$ void fraction. However, the mass flow errors are still considerable.

A key finding is that the results shown in fig. 3 are highly repeatable. Possible explainations for poor repeatability observed by Skea and Hall (1999) include transmitter hardware/software design, and/or flow-tube design features (e.g. split flow) which may lead to variations in twophase flow profile at the same nominal experimental point.

While it is highly desirable to have a complete physical model of two-phase flow effects, this remains a challenging long-term research goal. In this initial study a more limited aim has been set. This is to determine whether the highly repeatable two-phase flow response shown in Fig. 3 can be reliably predicted on-line by a neural network using only internally observed parameters.

More specifically, the research was limited to a 1 " flowtube in a horizontal alignment dealing with two-phase flow mixtures of air and water in the temperature range $0-40^{\circ} \mathrm{C}$. Prior observation had indicated that at lower flows, in horizontal orientation, phase separation may occur, causing characteristically different (and much larger) mass flow errors. Thus in this study, the mass flow rate was limited to the range 1.5 to $3.6 \mathrm{~kg} / \mathrm{s}$.

\section{NEURAL NETWORKS APPLIED TO THE CORIOLIS MASS FLOW METER}

In recent years neural networks have emerged as a practical technology, with successful applications in many fields, including pattern recognition, identification, classification, speech recognition and control systems (Bishop 1995). A feed-forward neural network provides a nonparametric framework for representing a nonlinear functional mapping between an input and an output space.

Of the various network models, the multi-layer perceptron (MLP) and the radial basis function (RBF) network have been used in this study.

It has been proved that an MLP with one hidden layer (each unit having a sigmoidal activation function) can approximate arbitrarily well any continuous mapping (Cybenko 1988, Hornik et al 1989). Hence, this type of network can be used to model the non-linear relationship between the mass-flow-error of the coriolis flow meter under two-phased flow and some of its internal parameters.

The set of network weights necessary for accomplishing the desired mapping is found in an optimisation process called training. During supervised training the network is repeatedly presented with the training set (a collection of input examples $\mathrm{x}_{\mathrm{i}}$ and their corresponding desired outputs $\mathrm{d}_{\mathrm{i}}$ ) and the weights are updated such that an error function is minimised. For the interpolation problem in hand, a suitable error function is the sum-of-square error, which for an MLP with one output is given by:

$$
\mathrm{I}=\sum_{\mathrm{i}=1}^{\mathrm{P}} \mathrm{e}_{\mathrm{i}}(\mathrm{t})^{2}=\sum_{\mathrm{i}=1}^{\mathrm{p}}\left(\mathrm{d}_{\mathrm{i}}-\mathrm{y}_{\mathrm{i}}\right)^{2}
$$

where $d_{i}$ is the target corresponding to input $\mathrm{x}_{\mathrm{i}}$; $\mathrm{y}_{\mathrm{i}}$ is the actual neural network output correspond to $\mathrm{x}_{\mathrm{i}}$;

$\mathrm{P}$ is the number of examples in the training set.

The other neural network architecture investigated is the RBF network. The RBF methods have their origins in techniques for performing exact interpolation of a set of data points in a multi-dimensional space (Powell 1987). A RBF network generally has a simple architecture consisting of two layers of weights, in which the first layer contains the parameters of the basis functions, and the second layer forms linear combinations of the activation of the basis functions to generate the outputs. This is achieved by representing the output of the network as a linear superposition of basis functions, one for each data point in the training set. In this form, training is faster than for a MLP (Bishop 1995).

Initially, it might appear that the RBF approach is more suited to the two-phase flow problem, particularly if it is viewed simply as interpolation between a database of previously recorded mass-flow errors. Indeed Luntta and Halttunen (1999) suggested RBF over MLP for a different measurement correction application.

The key disadvantage of the RBF network is that one node is required for each observation in the training set. For the two-phase flow problem this is likely to entail several hundred nodes, compared to less than twenty for the MLP. RBF 
is thus less appropriate for on-line implementation where storage and processing requirements must be considered.

During early experiments, several internally monitored parameters were observed to manifest significantly different behaviour in the presence of two-phase flow. At the outset, it was not clear which of these parameters (if any) could be used to predict the mass flow errors. The RBF approach was considered suitable for the rapid evaluation of new data and the assessment of which variables are contributory. The MLP network was used to give a compact solution suitable for real-time implementation.

The internal sensor parameters of interest are as follows:

Observed density The most widely used metric of two-phase flow is the void (or gas) fraction defined as the proportion of gas by volume. For example equation (1) used by Hemp and Sultan (1989) models the mass flow error given the void fraction. In practice, outside of a laboratory, on-line assessment of void fraction is rarely possible. However, for a coriolis mass flow meter, the reported process fluid density does provide an indirect measure of void fraction assuming the 'true' liquid density is known. This reported process density is subject to similar errors as the mass flow measurement in the presence of two-phase flow. However, experience has indicated that these errors are highly repeatable and that the drop in density is a suitable monotonic but non-linear indicator of void fraction, which can be monitored on-line within the flowmeter.

An alternative measure might be the resonant frequency of the flowtube, which, for this design, is related to the process fluid density by the following equation:

$$
\rho=\frac{\left(\mathrm{DC}_{1} \cdot \Delta \mathrm{T}+\mathrm{DC}_{2}\right)}{\mathrm{f}^{2}}+\mathrm{DC}_{3}+\mathrm{DC}_{4} \cdot \Delta \mathrm{T},
$$

where:

$\rho$ is the observed density

$\mathrm{DC}_{\mathrm{i}}$ are the density calibration coefficients;

$\Delta \mathrm{T}$ is the deviation from the calibration temperature;

$\mathrm{f}$ is the resonant frequency.

Although the resonant frequency is a fundamental property of the flowtube it has the disadvantage of dependency upon, amongst other factors, the flowtube size (via the calibration constants $\mathrm{DC}_{\mathrm{i}}$ ) and process temperature. Thus for two flowtubes of the same design but of different size, the same void fraction will produce different frequency shifts, whereas experimental data suggests that the density shift will be similar.

Knowledge of the 'true' single-phase liquid density can be learned on-line or configured by the user (possibly including a temperature coefficient). Both approaches have been implemented and appear satisfactory.

For the remainder of the paper, the drop in density will be used as the $\mathrm{x}$-axis parameter in graphs showing two-phase flow behaviour. Note that in the following 3D plots, which capture the results from 134 on-line experiments, a full range of density drop points is not possible at high flow rates due to air pressure limitations in the flow rig. Also, the effects of temperature, though not shown in the graphs, have been determined experimentally.

Damping Most coriolis meters use positive feedback to maintain flowtube oscillation. The sensor signals provide the frequency and phase of the flowtube oscillation, which are multiplied by a 'drive gain' to provide the currents supplied to the driver(s) (Henry et al, 2000):

$$
\mathrm{K}_{0}=\frac{\text { drive signal out (Amps) }}{\text { sensor signal in (Volts) }}=\frac{\mathrm{I}_{\mathrm{D}}}{\mathrm{V}_{\mathrm{A}}}
$$

Normally the drive gain is modified to ensure a constant amplitude of oscillation, and is roughly proportional to the damping factor of the flowtube.

One of the most characteristic features of twophase flow is a rapid rise in damping. For example, with normal operation a $25 \mathrm{~mm}$ flowtube has $\mathrm{V}_{\mathrm{A}}=0.3 \mathrm{~V}, \mathrm{I}_{\mathrm{D}}=10 \mathrm{~mA}$, and hence $\mathrm{K}_{0}=0.033$. With two-phase flow, values might be as extreme as $\mathrm{V}_{\mathrm{A}}=0.03 \mathrm{~V}, \mathrm{I}_{\mathrm{D}}=100 \mathrm{~mA}$, and $\mathrm{K}_{0}=3.3$, a hundred-fold increase. Figure 4 shows how damping varies with two-phase flow.

Sensor Balance In the coriolis flowmeter under consideration, feedback control is used to ensure that one of the sensor inputs has a fixed amplitude of oscillation. The other sensor amplitude is not constrained, and tends to vary from the other by up to $3 \%$, depending on manufacturing and operation conditions. The sensor balance is defined as: 


$$
\mathrm{Bs}=\frac{\text { amplitude }\left(\mathrm{V}_{\mathrm{A}}\right)}{\operatorname{amplitude}\left(\mathrm{V}_{\mathrm{B}}\right)}
$$

The sensor balance should thus be close to 1.0 for normal operation. Figure 5 illustrates how the sensor balance varies with aeration.

RPSS and CPSS The mass flow measurement is a function of the phase difference between the two sensor signals. As explained in (Henry et al, 2000), in the digital coriolis transmitter, a phase value is calculated for each complete cycle of data on each sensor signal. Phase difference is given by the weighted difference of these phase values.

On average, the phase value on a sensor signal should be zero. In practice, the average is very close to zero, but in the presence of aeration, this mean value changes, as shown in figs. 6 and 7. Note that although the changes are small in magnitude (e.g. no greater than $0.06^{\circ}$ ), the phase difference corresponding to full flow is only $4^{\circ}$, so these shifts are comparable to the magnitude of the two-phase flow errors in fig 3.

It is not clear what causes this deviation in the phase. In fact two measures of phase on the sensor signal are used. The RPSS (Raw Phase Sensor Signal) is the raw value. The CPSS (Corrected Phase Sensor Signal) is the sensor phase corrected for the instantaneous rate of change of amplitude for reasons explained in (Henry et al, 2000). As shown in figure 7, CPSS behaves significantly differently from the RPSS. Thus, both are included in the initial set of variables used to predict the mass flow error induced by two-phase flow.

Apparent flow rate and temperature As fig. 3 shows, the mass flow error varies with the (true) flow rate. Temperature variations have also been observed. Of course the true mass flow rate is not available within the transmitter itself when it is subject to two-phase flow, as that is the desired objective of the research. However the observed (faulty) flow rate, as well as the temperature, are candidates as input parameters to the neural network.

\section{NETWORK TRAINING AND ON- LINE CORRECTION OF MASS FLOW ERROR}

Implementing the neural network analysis for the mass flow measurement error prediction consists of the following steps:
- Training the neural network to recognise the mass flow error pattern in training experimental data;

- Testing the performance of the neural network on a new set of experimental data;

- On-line implementation of the neural network for measurement error prediction and correction.

The prediction quality of the neural network depends on the wealth of the training data. To collect the neural network training data, a series of two-phase (air-water) experiments were conducted on a rig shown in fig. 8. The flow loop includes a master meter (Micro Motion) the self-validating (SEVA) coriolis meter, and a diverter to transfer flow to a weigh scale. The SEVA coriolis meter has a totalisation function which can be triggered by external signals. The rig control is arranged so that both the flow diverter (supplying the weighscale) and coriolis totalisation are triggered by the master meter at the beginning of an experiment, and again after the master meter has observed $100 \mathrm{~kg}$ of flow. The weigh scale total is used as the reference to calculate mass flow errors by comparison with the totalised flow from the digital transmitter, the master meter acting as an additional check. The uncertainty of the experimental rig is estimated at about $0.1 \%$ on typical batch sizes of $100 \mathrm{~kg}$. For single phase experiments the digital transmitter delivers mass flow totals within $0.2 \%$ of the weigh scale total (Henry et al., 2000). For two-phase flow experiments, air is injected into the flow after the master meter and before the SEVA meter. At low flow rates, density drops of up to $30 \%$ are achieved. At higher flow rates, at least $15 \%$ density drops are achieved.

At the end of each batch the coriolis meter reports the batch average of each of the following parameters: temperature, damping, sensor balance, density, RPSS, CPSS, flow rate, along with the total (uncorrected) flow. These parameters are thus available for use as the input data of a neural network.

The output or the target of the neural network is the mass flow error in percentage calculated as:

mass_error $\%=\frac{\text { coriolis }- \text { weighscale }}{\text { weighscale }} \times 100$

Figure 9 shows how the mass flow error varies with flow rate and density drop. Although the general trend follows the bubble model, there are some interesting features. For example, for high flow rates and low density drops, the mass 
flow error becomes slightly positive (by as much as $1 \%$ ): the bubble model only predicts a negative error. Clearly, in this region of the experimental space, and for this flowtube design, some other physical process is taking place to overcome the missing mass effects of two-phase flow.

At the outset of neural net training, many design issues were unclear. Seven parameters were selected as possible inputs to the neural network, on the basis of their 'interesting' behaviour in response to two-phase flow. It was not clear however which of them would actually provide more information than noise to the network. Another issue was whether to use a simple heuristic or theoretical model (e.g. the bubble model) to make a basic prediction, and then use a neural net to predict the residual errors. To provide a quick evaluation of different designs, the RBF network structure was used. Following standard procedure, the prediction performance of each model was compared using a validation data set, independent of the training set.

After various alternatives were explored, the best results were obtained using only four input parameters: temperature, damping, density drop and apparent flow rate. This is intuitively appealing, as each of these relate to basic physical parameters. The sensor balance, RPSS and CPSS factors, which in any case show considerable variation between flowtubes, do not appear to offer any additional information, despite their intriguing behaviour. Less satisfactory perhaps is the result that the best fit was achieved using the neural net on its own, rather than as a correction to the bubble model or a simple curve fit.

Although an RBF network is quickly trained, an on-line implementation is relatively expensive in terms of computational and data storage requirements. Hence a MLP neural network was used for on-line implementation. Comparisons between RBF and MLP networks with the same data sets and inputs have shown broadly similar performance on the test set. It is thus reasonable to assume that the input set delivering the best RBF design will also deliver a good (if not the best) MLP design. MPL nets were trained using the scaled conjugate gradient algorithm. The facilities of the Neural Network Toolbox of the Matlab package were used for network training. After further design options were explored, the best performance came from an 4-9-1 MLP having as inputs the temperature, damping, drop in density and flow rate, along with the mass flow error as output.
Against a validation set, the best network provided mass flow rate predictions to within $2 \%$ of the true value. However, the real challenge is to demonstrate that the neural network can match this performance when implemented on-line. This proved to be straightforward. Routines for the detection and correction for two-phase flow have been coded and incorporated into the digital coriolis transmitter. Figure 10 shows the (residual) mass-flow error when corrected on-line over 134 new experiments. All errors are within 2\%, and most are considerably less. The random scatter is due primarily to residual error in the neural net correction algorithm (as stated earlier, the uncertainty of the flow rig is $0.1 \%$ ). Any obvious trend in the data would imply scope for further correction. These errors are of course for the average corrected mass flow rate (i.e. over a batch).

Figure 11 shows how the on-line detection and correction for two-phase flow is reflected in the SEVA interface generated for the mass flow measurement. (Henry and Clarke, 1993). In the graph the lower, continuous line is the raw mass flow rate, the measurement surrounded by the uncertainty band is the corrected or validated measurement, and the dashed line is the mass flow rate from the master meter, which is positioned prior to the air injection point (fig. 8).

With single-phase flow (up to $t=5 \mathrm{~s}$ ), the massflow measurement is CLEAR and it has a small uncertainty of about $0.2 \%$ of reading. Once twophase flow is detected, the neural network correction is applied every interleaved cycle (i.e. at $180 \mathrm{~Hz}$ ), based on the values of the internal parameters averaged (using a moving window) over the last second. During two-phase flow, the measurement status is set to BLURRED, and the uncertainty increases to reflect the reduced accuracy of the corrected measurement. The uncorrected measurement (lower dark line) shows a large offset error of about $30 \%$.

The master meter reading agrees to a first approximation with the corrected mass flow measurement. The apparent delay in its response to the incidence of two phase flow is attributable to communication delays in the rig control system, and its squarewave-like response it due to the control system update rate of only once per second. Both the raw and corrected measurements from the digital transmitter show a higher degree of variation than with single phase flow. There is of course 
some difficulty in defining and measuring what the instantaneous 'true' mass flow is. The master meter reading gives a useful measure of the water phase entering the two-phase zone, and there is a clear similarity between the master meter reading and the 'average' corrected reading. However, plug flow and air compressibility within the complex 3-D geometry of the flowtube may result in not only flow rate variations, but with the instantaneous mass flow in being different from the mass flow out. Further research, and in particular, comparision of results using a straight flowtube, would be instructive.

With the SEVA approach the measurement is not simply labeled good or bad by the sensor. Instead, if a fault occurs, a correction is applied as far as possible and the quality of the resulting measurement is indicated through the BLURRED status and increased uncertainty. The user can thus assess application-specific requirements and options in order to decide whether to continue operation with the reduced quality of the corrected mass-flow rate, to switch to an alternative measurement if available, or to shut down the process. If twophase flow is present only during part of a batch run (e.g. at beginning or end), then there will be a proportionate weighting given to the uncertainty of the total mass of the batch.

\section{CONCLUSIONS}

The inability of coriolis mass flow meters to deal with two-phase flow has limited their application. Previous work at Oxford has demonstrated that it is possible through careful transmitter design to prevent flowtube shutdown. In this paper it has been shown that for one flowtube design at least, the use of a robust transmitter provides a repeatable twophase flow response which is thus amenable to mass flow correction. Although the basic trend follows the 'bubble model' there are sufficient differences to suggest that more detailed modelling is required. As a demonstration of the viability of compensation, a neural networkbased solution has been implemented on-line, which reduces mass flow errors from as high as $20 \%$ to within $2 \%$, for a limited range of flow, temperature and void fractions. The detection and compensation for two-phase flow readily fits into the SEVA framework for the reporting of on-line measurement quality.

Clearly it would be desirable to gain further insight into two-phase flow effects, over a broader range of experimental parameters, through improved physical modelling. Whilst the bubble model predicts the general trend of the mass flow errors for medium and high flows, it does not explain the positive errors observed at high flows and low density drops, not does it account for the variations in mass flow error with damping, flow rate and temperature. One possible criterion for measuring the predictive power of a new physical model would be to compare its performance against that of a neural net. As noted earlier, it is disappointing that a neural net on its own outperformed the bubble model, even with a neural net to correct for the residual errors. One complicating factor in the current work is the complexity of the flowtube design. Possibly a straight flowtube (together with a suitably robust transmitter) would assist in providing closer correlation between theory and experiment.

Several practical benefits would accrue from improved modelling and correction of twophase flow effects in coriolis meters. These include extending their range to applications where two-phase flow occurs on an occasional or continuous basis, and replacing more expensive two-phase instrumentation.

\section{REFERENCES}

Bishop, C. M.(1995), Neural Networks for Pattern Recognition, Oxford University Press.

Demuth, $\mathrm{H}$ and Beale, M, Neural Network Toolbox for use with Matlab, The MathWorks, Inc, 1998

Grumski, J. T. and Bajura, R.A (1984). Performance of a coriolis-type mass flow meter in the measurement of twophase(air-liquid) mixtures. ASME Fluid Engineering Division Publication FED Vol. 17, pp75-83.

Hemp J. And G. Sultan (1989), On the theory and performance of Coriolis Mass Flowmeters. Proceedings of the International Conference on Mass Flow Measurement Direct and Indirect, IBC Technical Services, London, Feb 1989.

Henry, M. P. and D.W. Clarke (1993), The SelfValidating Sensor: Rationale, Definitions and Examples. Control Engineering Practice, 1 (4), pp.585-610.

Henry, M. P. (1995a), A Self-Validation Coriolis Meter. Control Engineering Practice, 42 (6), pp. 81-86. 
Henry, M. P. (1995b), Fieldbus and Sensor Validation. IEE Computing and Control Journal, 6 (6), pp. 263-272.

Henry M. P. (1998), Summary of Aeration Research, Internal Report, Department of Engineering Science, Oxford University, U.K.

Henry, M. P, Clarke, D. W., Archer, N., Bowles, J., Leahy, M. J., Liu, R. P., Vignos, J., Zhou, F. B.(2000). A selfvalidating digital coriolis mass-flow meter: an overview. Control Engineering Practice, Vol. 8, No. 5, pp487-506.

Hunt, K. J., D. Sbarbaro, R. Zbikowski and P. J. Gawthrop (1992), Neural Network for Control Systems - A survey. Automatica, Vol. 28, No 6 pp. 1083-1112

Luntta, E. and Halttunen, J. (1999). Neural network approach to ultrasonic flow measurements. Flow Measurement and Instrumentation, Vol. 10, pp35-43

Rumelhart, D. E., G. E. Hinton and R. J. Willians (1986), Learning internal representations by error propagation. In D. E. Rumelhart and J. L. McClelland (Eds.) Parallel Distributed Processing. MIT Press. Cambridge, MA.

CybenKo, G. (1988), Continuous valued networks with two hidden layers are sufficient. Technical Report, Department of Computer Science, Tufts University.

Hornik, K., M. Stinchcombe and H. White (1989), Multilayer feedforward networks are universal approximators. Neural Networks, 2, pp. 359-366.

Powell, M. J. (1987). Radial basis functions for multivariable interpolation: a review. In J. C. Mason and M. G. Cox (Eds.), Algorithms for Approximation, pp. 143167. Oxford: Clarendon Press.

Riedmiller, M. and H. Braun (1993), A direct adaptive method for faster backpropagation learning: The RPROP algorithm. Proceedings of the IEEE International Conference on Neural Networks.

Reimann, J. Developments in two-phase mass flow rate instrumentation.

Skea, A. F. and Hall, A. R. W (1999), Effects of gas leaks in oil flow on single-phase flowmeters. Flow Measurement and Instrumentation, Vol. 10, pp146-150. 


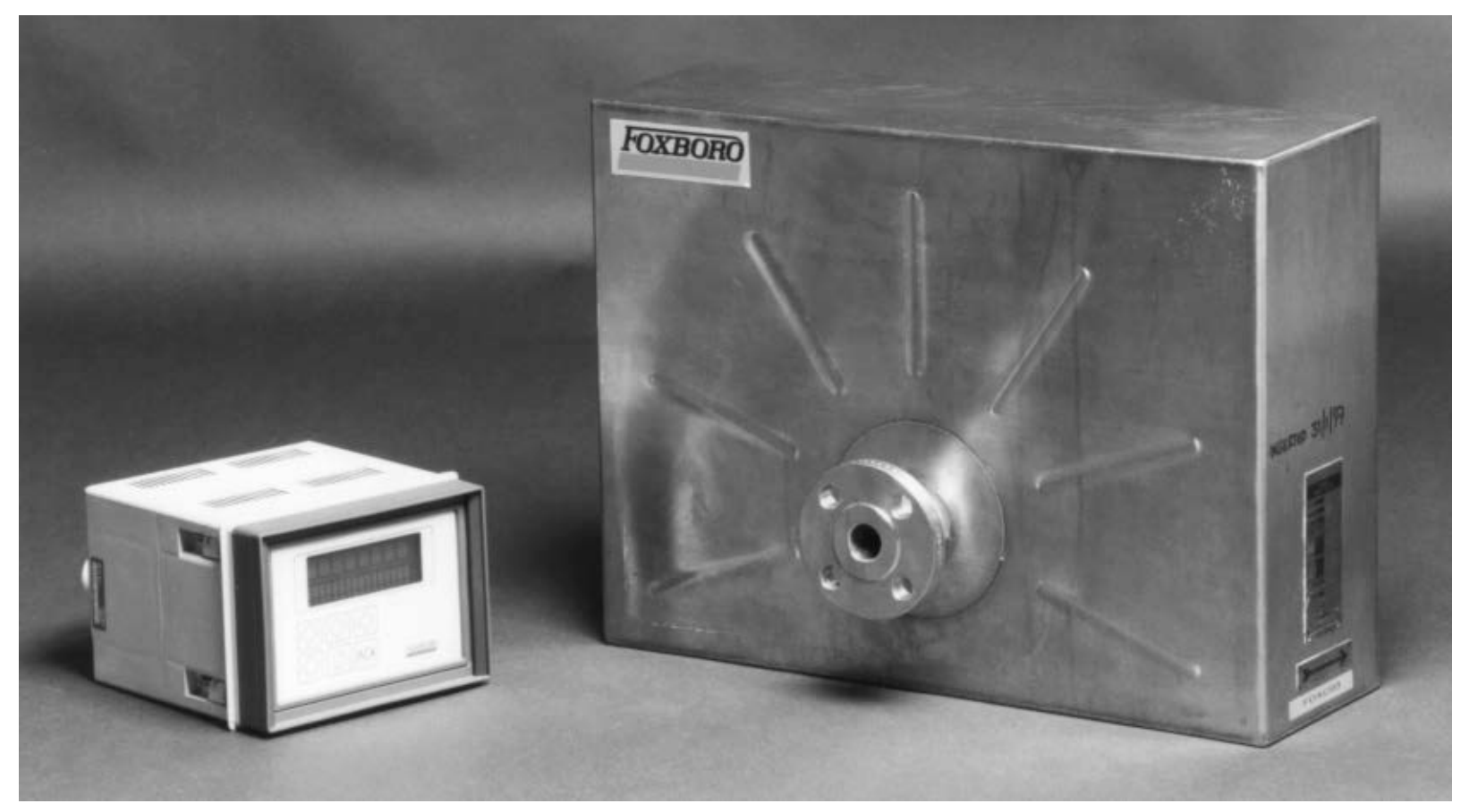

Figure 1. A commercial Coriolis mass-flow meter, with transmitter (left) and flowtube (right) (Reproduced from Henry et al., 2000). 


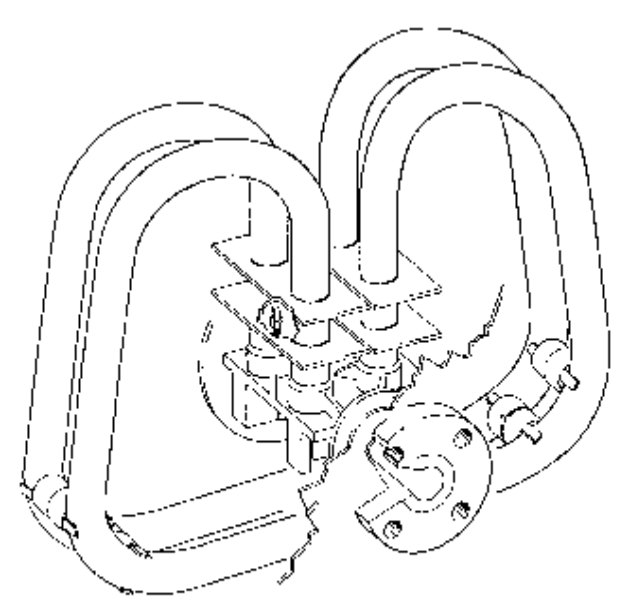

Flowtube

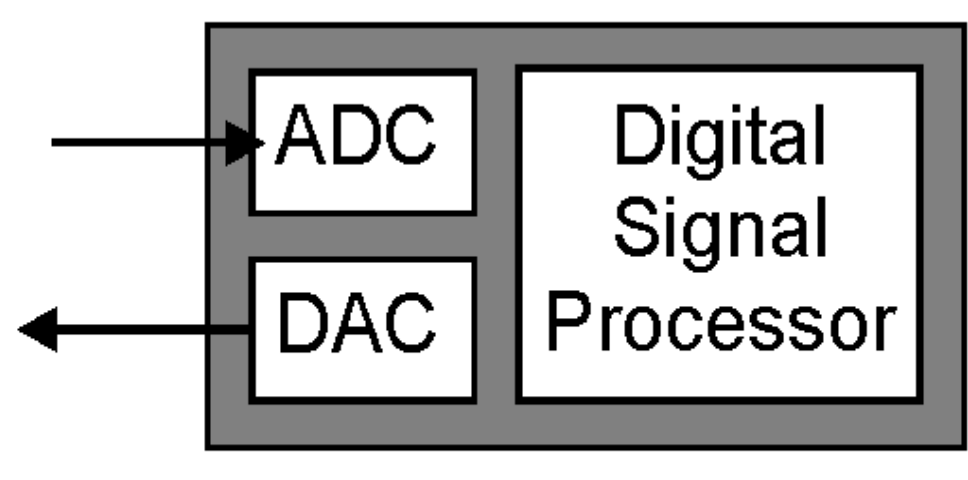

Digital Transmitter

Figure 2 Digital Coriolis flow meter (Reproduced from Henry et al., 2000). 


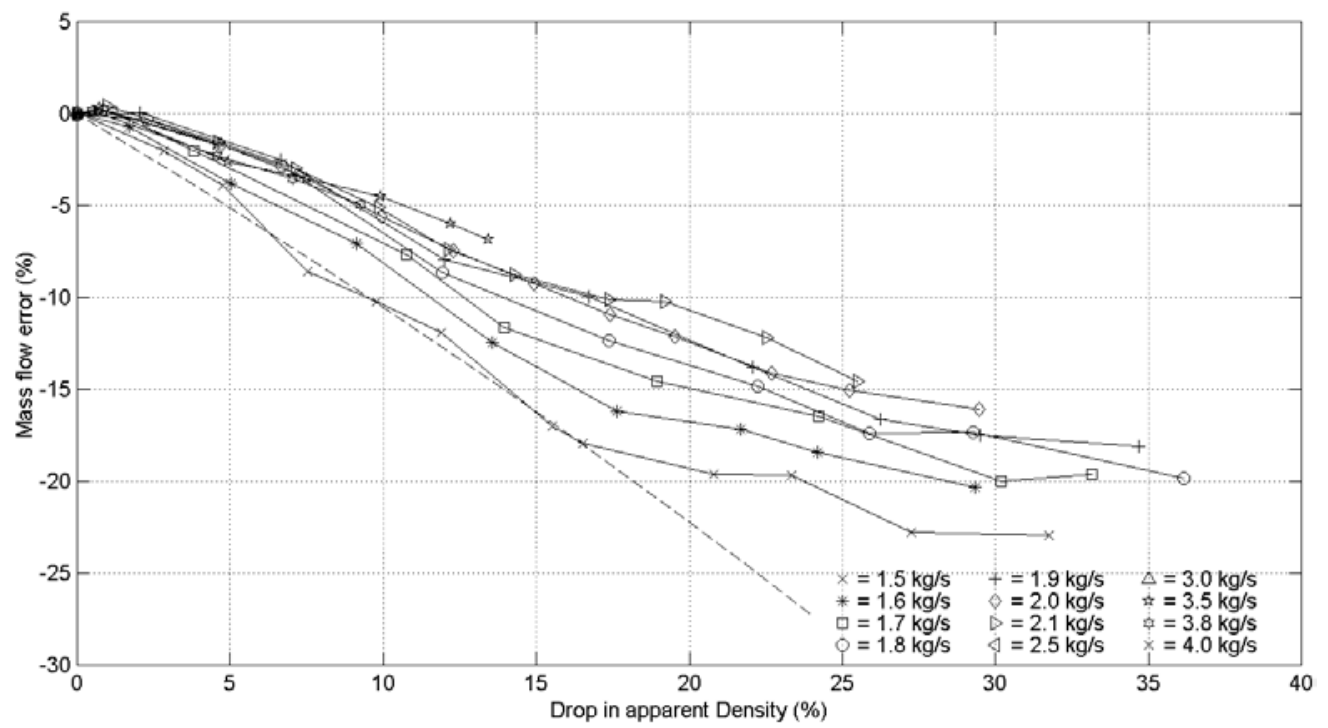

Figure 3 Comparison of experiment results with the prediction of 'bubble' model (Reproduced from Henry et al., 2000). 


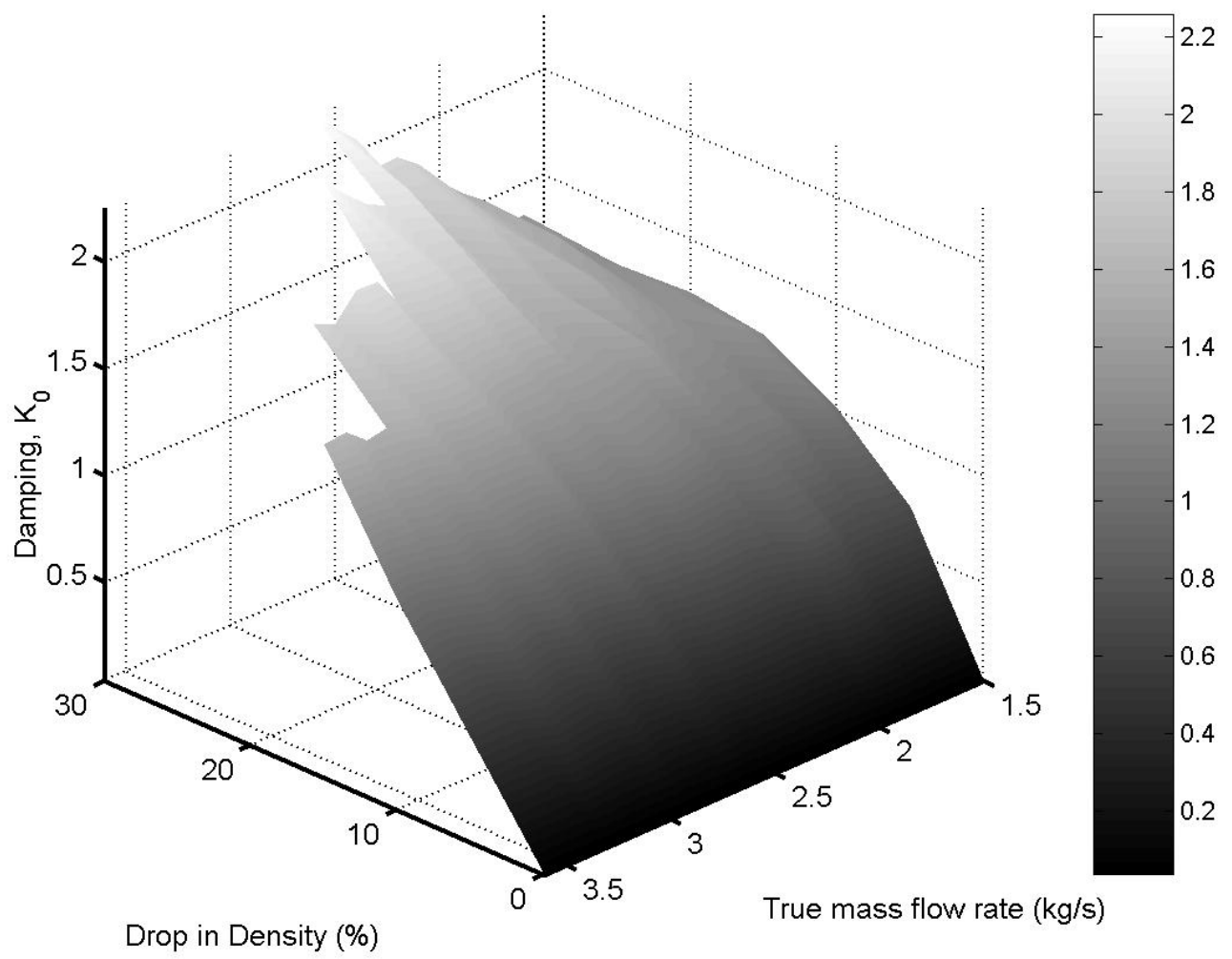

Figure 4 Damping changes under two- phase flow 


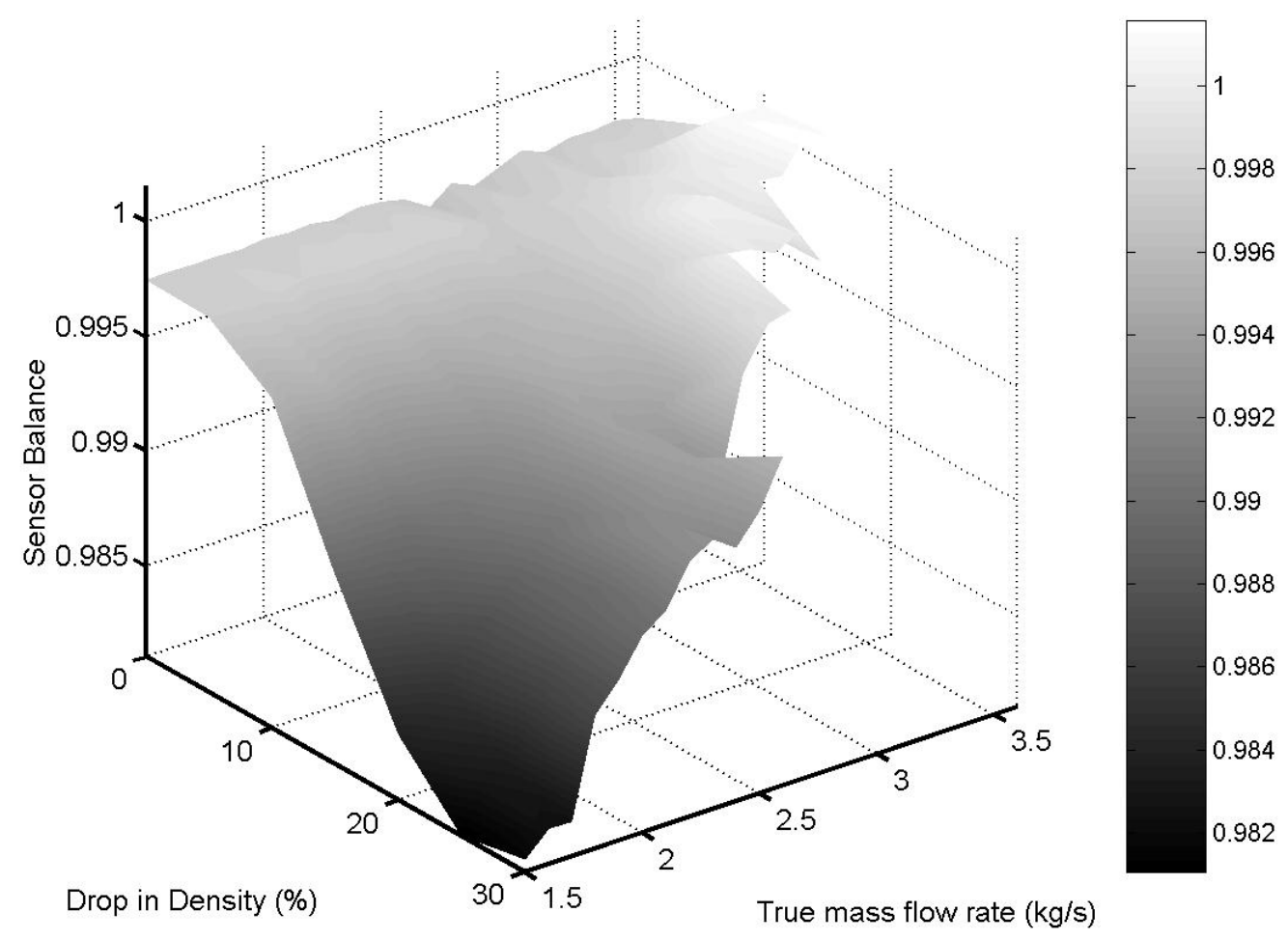

Figure 5 Sensor balance under two-phase flow conditions 


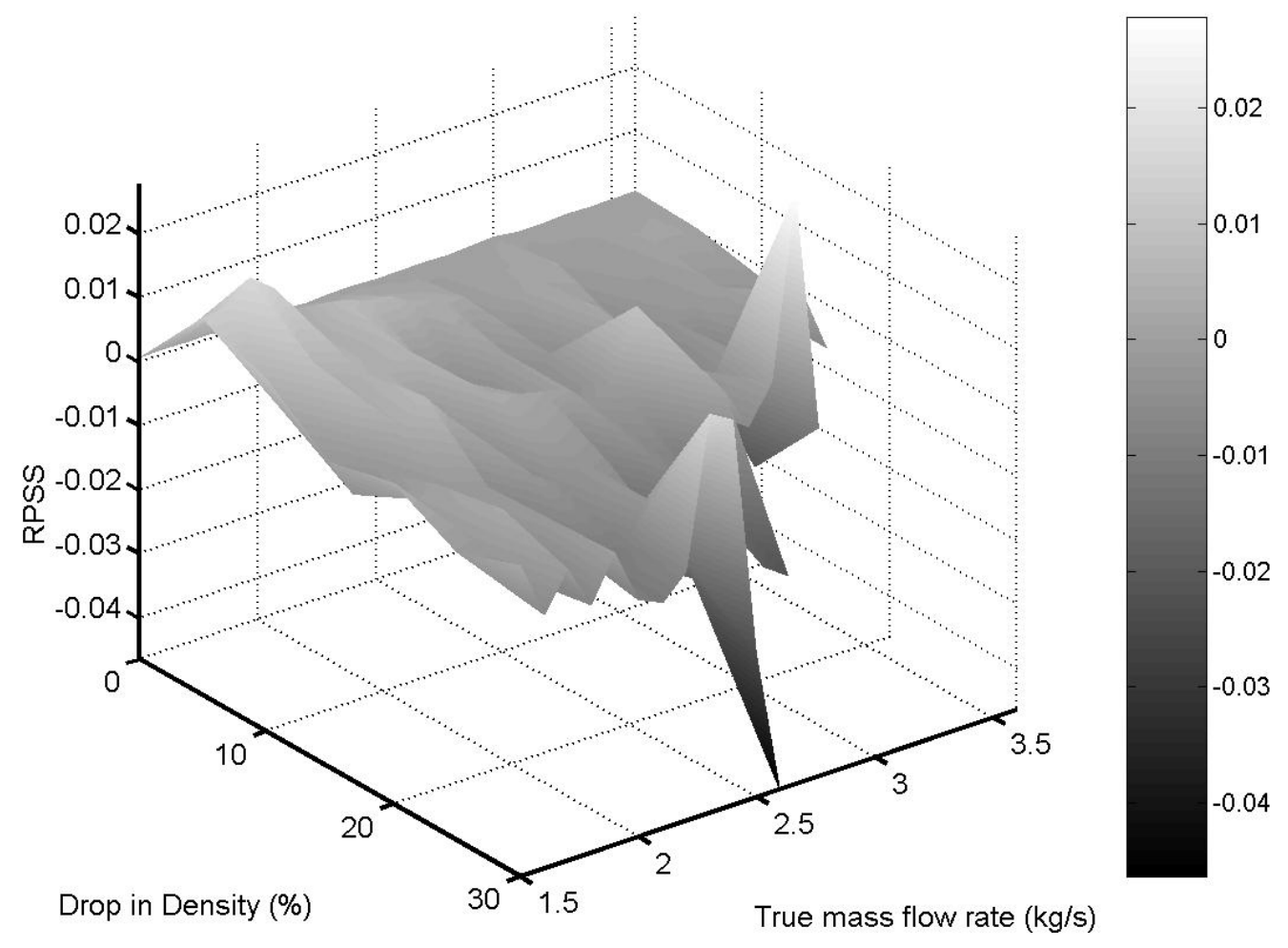

Figure 6 RPSS changes under two-phase flow conditions 


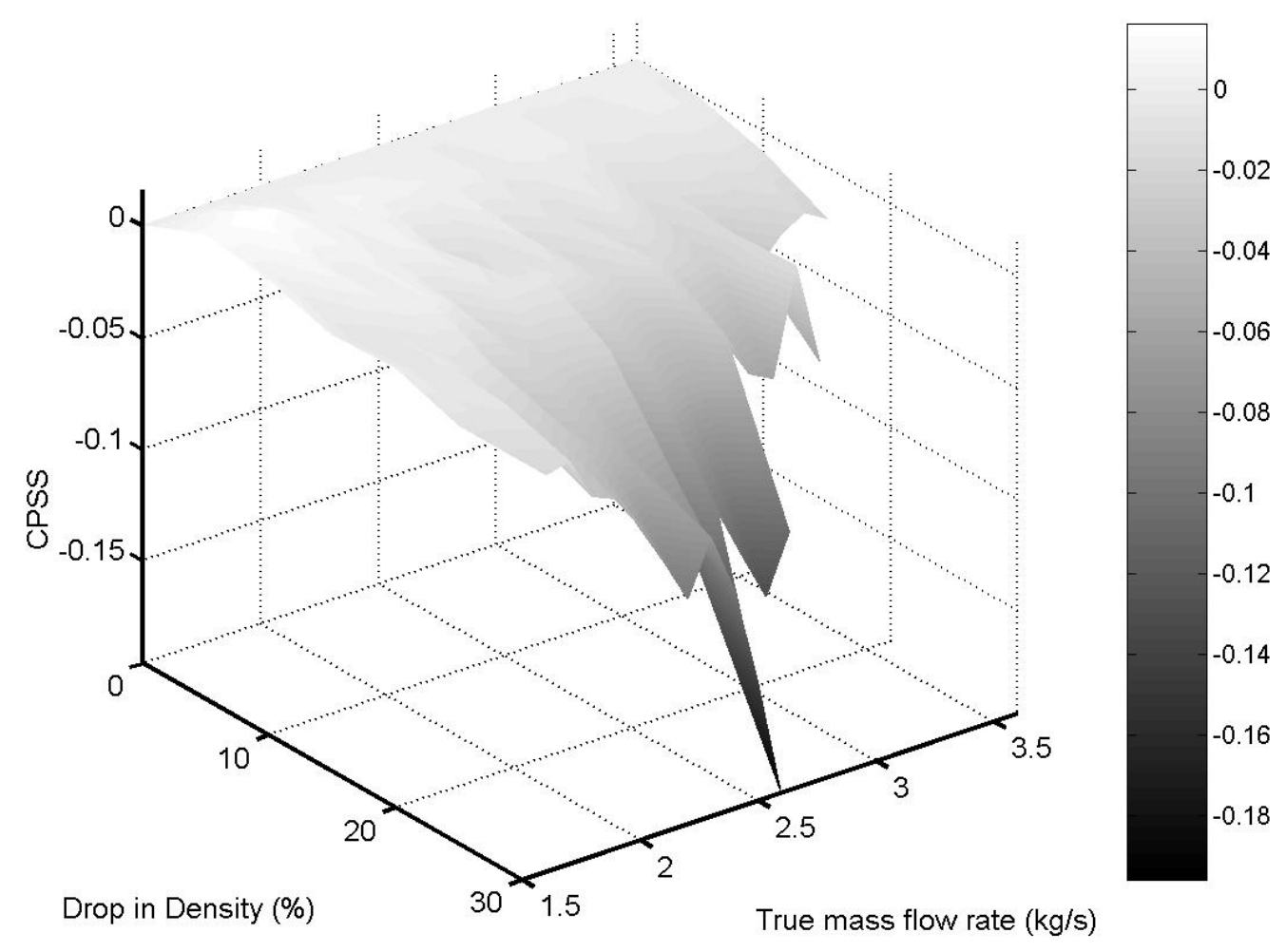

Figure 7 CPSS changes under two-phase flow conditions 


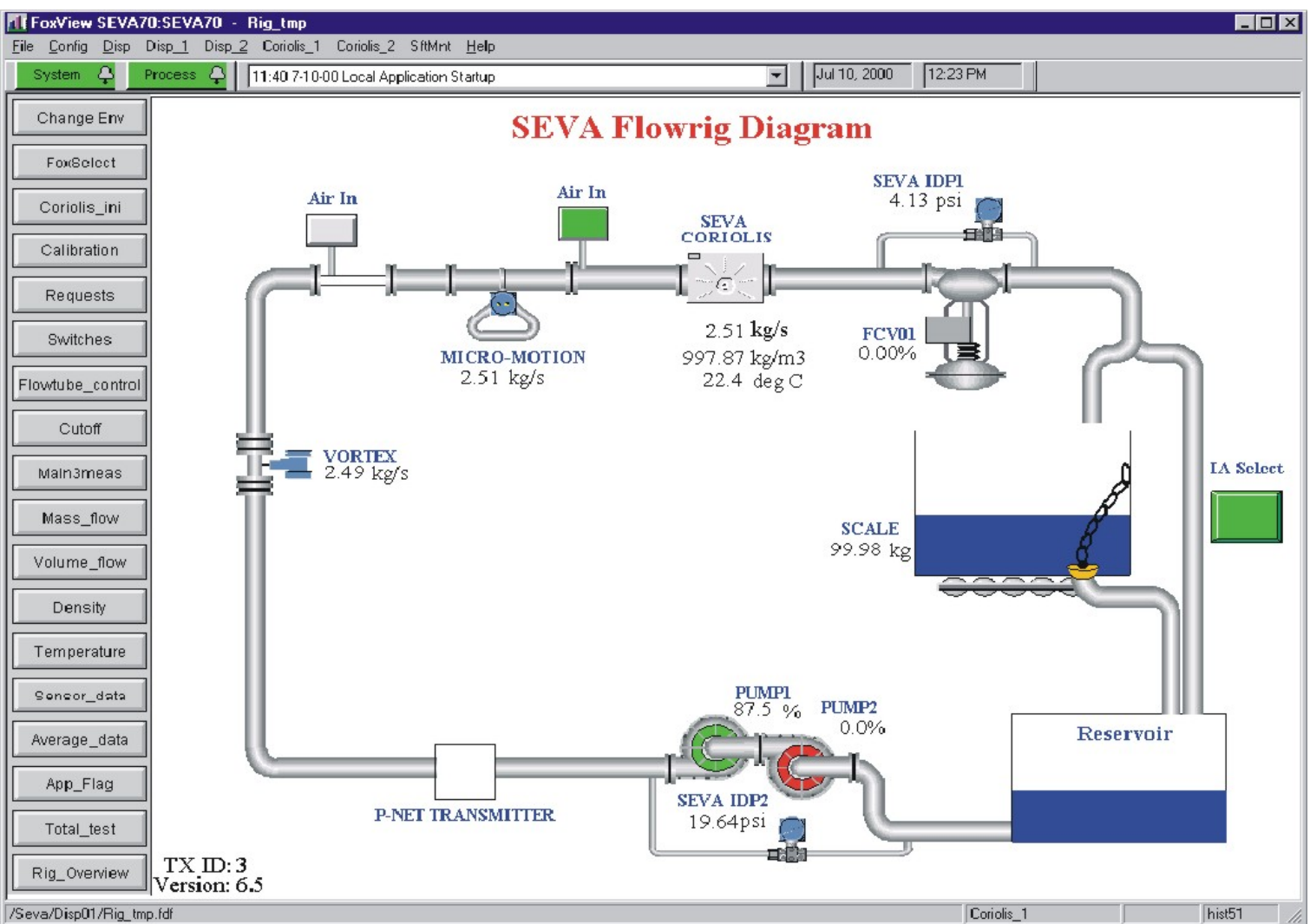

Figure 8 Screen dump showing experimental flow rig 


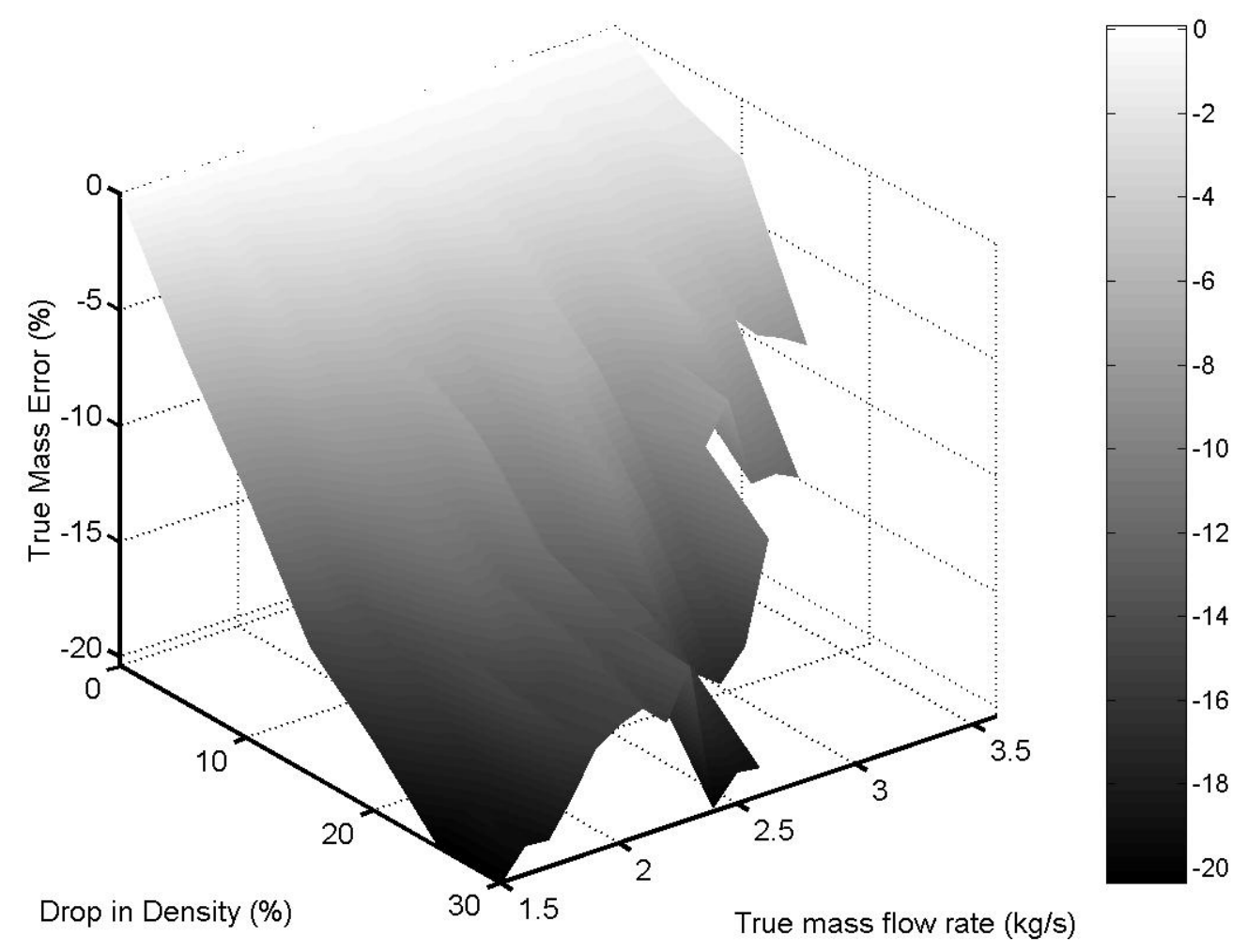

Figure 9 True mass flow error with two-phase flow 


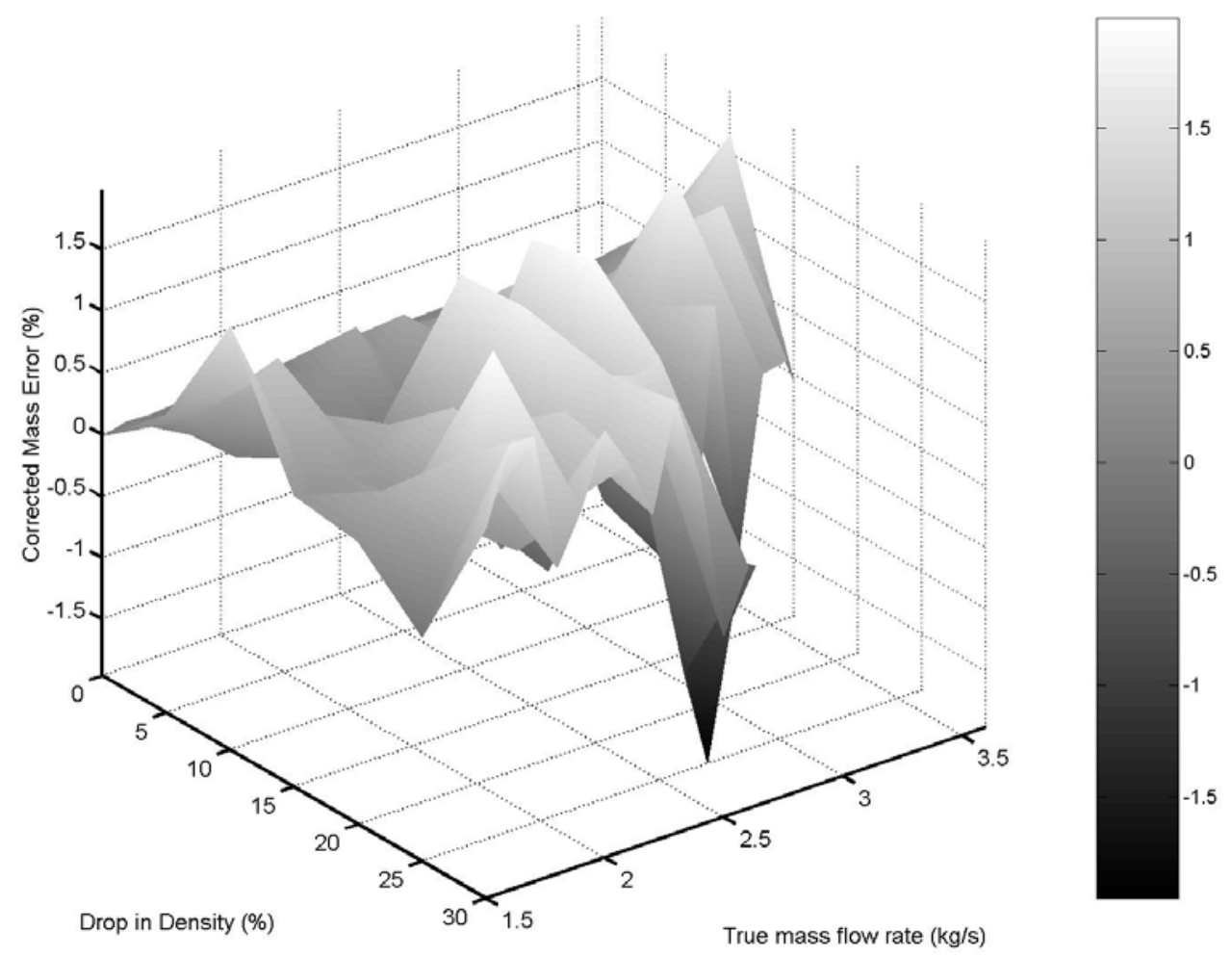

Figure 10 Corrected mass flow error with two-phase flow 


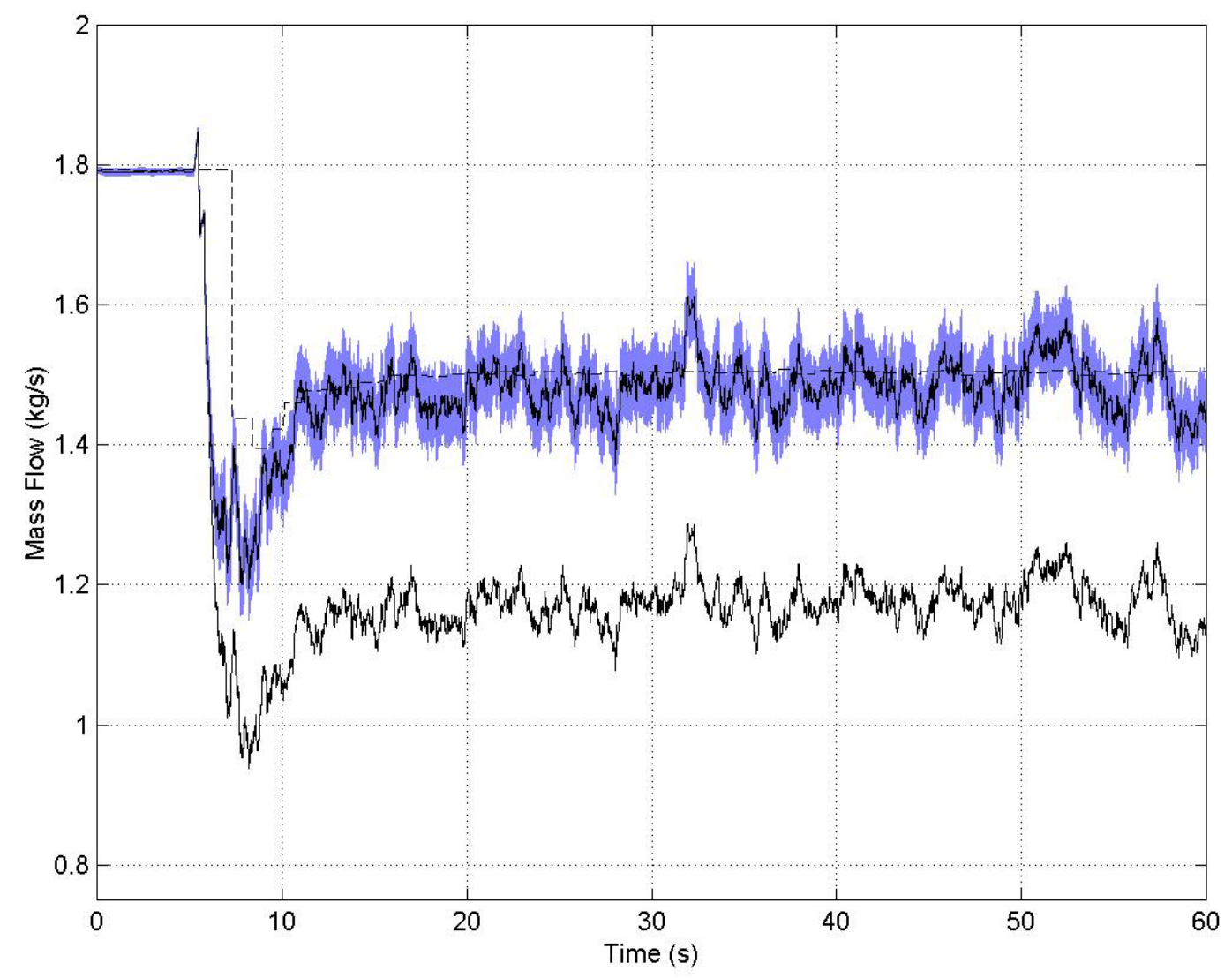

Figure 11 On-line response of self-validating digital transmitter to the onset of two-phase flow. Lower line: uncorrected mass-flow. Upper dark line: corrected mass-flow. Dashed line: reading from master meter positioned prior to air injection point. 